\title{
Fallopian Tube Gestational Choriocarcinoma
}

National Cancer Institute

\section{Source}

National Cancer Institute. Fallopian Tube Gestational Choriocarcinoma. NCI Thesaurus.

Code 6278

A malignant trophoblastic tumor that arises from the fallopian tube during pregnancy. 